\title{
Efectividad De Un Polímero Retenedor De Humedad Para Elevar La Supervivencia En Plantaciones De Mezquite (Prosopis Glandulosa Torr.) En Zonas Áridas
}

\author{
Gabriel Sosa Pérez (PhD) \\ David Efraín Hermosillo Rojas (MS) \\ Pedro Jurado Guerra (PhD) \\ Manuel Pomposo Alarcón Bustamante (MS) \\ José Luis García Pérez (MS) \\ Instituto Nacional de Investigaciones Forestales Agrícolas y Pecuarias \\ (INIFAP), Campo Experimental "La Campana" Km. 33.3 Carretera \\ Chihuahua-Ojinaga. Aldama, Chihuahua, México
}

Doi:10.19044/esj.2021.v17n7p55

Submitted: 15 December 2020

Accepted: 12 February 2021

Published: 28 February 2021
Copyright 2021 Author(s)

Under Creative Commons BY-NC-ND

4.0 OPEN ACCESS

Cite As:

Sosa Pérez G., Hermosillo Rojas D.E., Jurado Guerra P., Alarcón Bustamante M.P. \& García Pérez J.L. (2021). Efectividad De Un Polímero Retenedor De Humedad Para Elevar La Supervivencia En Plantaciones De Mezquite (Prosopis Glandulosa Torr.) En Zonas Áridas. European Scientific Journal, ESJ, 17(7), 55. https://doi.org/10.19044/esj.2021.v17n7p55

\section{Resumen}

El uso de polímeros retenedores de humedad es una técnica que se ha utilizado para incrementar la supervivencia en plantaciones de restauración forestal, sin embargo, escasos estudios han evaluado su efectividad. El objetivo del estudio fue evaluar el efecto de diferentes dosis de un polímero retenedor de humedad en la supervivencia de tres plantaciones de mezquite (Prosopis glandulosa Torr.), en dos sitios de zonas áridas del estado de Chihuahua, México. Se utilizó un polímero hecho a base de potasio en dosis desde tres hasta diez gramos por planta. Se midió la precipitación en cada sitio, la humedad del suelo en cada planta y los porcentajes de supervivencia de cada plantación en diferentes fechas a lo largo de un año. Los porcentajes de supervivencia después de un año fueron $34 \%, 59 \%$ y $83 \%$. No se encontró efecto positivo de ninguna dosis de hidrogel en la supervivencia. La humedad en el suelo por efecto del polímero es muy corta en condiciones de poca precipitación y altas temperaturas, y los eventos de lluvia distanciados en 
tiempo no son suficientes para la rehidratación del polímero. Esto genera una igualdad de condiciones en términos de humedad del suelo para las diferentes dosis durante los meses críticos de sequía, en los cuales se registró la mayor mortandad de planta.

Palabras-claves: Hidrogel, Lluvia Sólida, Poliacrilato, Potasio, Restauración Forestal

\title{
Effectiveness of a Moisture-Retaining Polymer to Increase Survival in Mesquite Plantations (Prosopis Glandulosa Torr.) of Arid Lands
}

\author{
Gabriel Sosa Pérez (PhD) \\ David Efraín Hermosillo Rojas (MS) \\ Pedro Jurado Guerra (PhD) \\ Manuel Pomposo Alarcón Bustamante (MS) \\ José Luis García Pérez (MS)
}

Instituto Nacional de Investigaciones Forestales Agrícolas y Pecuarias

(INIFAP), Campo Experimental "La Campana" Km. 33.3 Carretera

Chihuahua-Ojinaga. Aldama, Chihuahua, México

\begin{abstract}
Moisture-retaining polymers are frequently used to increase survival in forest restoration plantations, however, few studies have evaluated their effectiveness. The objective of the study was to evaluate the effect of different doses of a moisture-retaining polymer on the survival of three mesquite plantations (Prosopis glandulosa Torr.), in two sites of arid lands in the State of Chihuahua, Mexico. A polymer made from potassium was used in doses from three to ten grams per plant. Precipitation at each site was measured, as well as soil moisture at each plant, and the survival percentages at different dates after plantations. The survival percentages in the three plantations after one year were $34 \%, 59 \%$, and $83 \%$. No positive effect of any dose was found on survival. The effect of the polymer on soil moisture is very short under low precipitation and high temperatures. Isolated rainfall events did not rehydrate the polymer. The highest plant mortality was during the critical months of drought, and soil moisture was the same for the different doses during these months.
\end{abstract}

Keywords: Forest Restoration, Hydrogel, Polyacrylate, Potassium, Solid Rain, Drough 


\section{Introducción}

El éxito o fracaso de una plantación de restauración forestal depende de un gran número de factores tanto climáticos como técnicos (RodríguezTrejo, 2006). Los primeros no se pueden controlar por el hombre, mientras que los factores técnicos tienen un origen totalmente antropogénico, y por ende pueden ser de alguna manera manipulados. Estos factores comprenden desde el sistema de producción de planta en el vivero, transporte, método de plantación y manejo post plantación (Capó, 2001). Dentro de estos factores, algunos se han considerado tradicionalmente muy importantes, como la calidad de planta (Mexal \& Landis, 1990; Prieto et al., 2003; Rodríguez, 2008). Sin embargo, aún y cuando se utilice planta de buena calidad y se prepare el suelo adecuadamente, existe otra causa muy importante de mortandad, la cual ha sido asociada principalmente a la sequía, especialmente en las zonas áridas y semiáridas del norte de México (Comisión Nacional Forestal [CONAFOR], 2016). Esto y otros factores han sido la causa de que en México se tengan bajos porcentajes de supervivencia. En 2014, por ejemplo, la supervivencia promedio a nivel nacional fue de $56 \%$ para todos los tipos de ecosistemas, y este valor fue mayor en más de cinco puntos porcentuales con respecto a los registrados en el periodo 2010-2013 (CONAFOR, 2016). Para las zonas áridas y semiáridas del estado de Chihuahua los mismos informes reportan valores de supervivencia de tan solo el $33 \%$.

Una de las técnicas que se ha utilizado en los últimos años como una medida para incrementar los porcentajes de supervivencia, es el uso de polímeros retenedores de humedad, comúnmente conocidos como hidrogeles. Los hidrogeles, hidro-retenedores o súper absorbentes, son polímeros hidrófilos hechos a base de acrilato de potasio, poliacrilato de potasio, o poliacriláminas que por su estructura molecular atraen magnéticamente las moléculas del agua logrando absorber entre 200 a 400 veces su peso en agua (Cisneros-Zayas et al., 2018; Freitas et al., 2002; Kazanskii \& Dubrovskii, 1992). Esta propiedad ha llevado a muchas aplicaciones prácticas, particularmente en la agricultura, para mejorar la retención de agua de los suelos y el suministro de agua de las plantas (Kazanskii \& Dubrovskii, 1992).

El tema de hidrogeles en el campo de la agricultura no es nuevo, sobre todo a nivel internacional, dado que existe un considerable número de estudios en relación a su efectividad en diferentes cultivos agrícolas (Al-Humaid \& Moftah, 2007; Rivera-Hincapié et al., 2007; Rojas et al., 2004), suelos (AbediKoupai et al., 2008; Andry et al., 2009; Cortés et al., 2007), o bajo diferentes climas y condiciones de humedad (Bai et al., 2010; Rojas et al., 2004). Sin embargo, en el sector forestal de México es un tema relativamente reciente con escasos estudios que han evaluado su efectividad. La literatura en el ámbito forestal internacional reporta una considerable variabilidad en la 
efectividad para reducir el estrés hídrico, pues mientras unos estudios reportan resultados positivos en el crecimiento o supervivencia (Al-Humaid \& Moftah, 2007; Sarvas et al., 2007; Viero et al., 2002; Viero \& Little, 2006), otros estudios sugieren que la aplicación no tiene efectos significativos en dichas variables (Del Campo et al., 2011; García, 2017; Patricio, 2014). Una reciente compilación de resultados de 42 estudios publicados en revistas científicas de revisión por pares hecha por Crous (2017), indicó que la efectividad del hidrogel en plantaciones forestales puede ser influenciado por: 1) químicos presentes en el suelo; 2) dosis aplicada; 3) tipo de polímero; 4) distribución del tamaño de partícula en el hidrogel; 5) textura del suelo; 6) restricciones físicas para la expansión del hidrogel; 7) capacidad hidrofílica del hidrogel; 8) conductividad hidráulica entre el suelo y las partículas del hidrogel bajo condiciones no saturadas; 9) el método de aplicación; y 10) la humedad del suelo al momento de la plantación y los riegos subsecuentes. Dada esta complejidad, no ha sido fácil encontrar recomendaciones o estudios en el ámbito de restauración forestal, que aseguren el éxito en la aplicación de hidrogel, especialmente para elevar la supervivencia en campo, que es la variable de mayor interés para técnicos o productores forestales.

Otro tema de vital importancia en la toma de decisiones para el uso de hidrogel es el aspecto económico. El costo del producto en el mercado mexicano al momento de este estudio varía entre $\$ 7.00$ a $\$ 14.00$ dólares estadounidenses por kilogramo, dependiendo de la calidad o marca. La cantidad a utilizar depende de la dosis recomendada, la cual tampoco está claramente establecida, pues mientras algunas empresas vendedoras recomiendan alrededor de $20 \mathrm{~g}$ por planta, esto puede resultar en costos muy altos para plantaciones de restauración forestal. Aunado al costo del producto además se agrega el costo de aplicación, dado que la aplicación de hidrogel implica: 1) transporte de agua al sitio de plantación (lo cual puede ser complicado en sitios de difícil acceso o escaza disponibilidad de agua); 2) hidratación del polímero en grandes contenedores; 3) transporte del hidrogel hidratado hasta cada planta a ser establecida; y 4) mezcla del polímero hidratado con el suelo que rodea a la raíz. Dadas todas estas implicaciones es importante realizar evaluaciones en campo, bajo condiciones reales que permitan generar información sobre el efecto de dichos retenedores en la supervivencia, lo cual es de vital importancia para las diferentes condiciones ambientales y en específico para las zonas áridas y semiáridas.

El objetivo general del estudio fue evaluar el efecto de un polímero retenedor de humedad en la supervivencia de tres plantaciones de mezquite (Prosopis glandulosa Torr.), en dos zonas áridas del estado de Chihuahua. Los objetivos específicos fueron evaluar: 1) el efecto de diferentes dosis de hidrogel en la humedad del suelo y supervivencia; y 2) el efecto del hidrogel bajo diferentes escenarios de precipitación. 


\section{Materiales y métodos \\ Áreas de estudio y establecimiento de las plantaciones}

La primera plantación se estableció en el municipio de Nuevo Casas Grandes, Chihuahua. El sitio se localiza en las coordenadas $30^{\circ} 50^{\prime} 35.55^{\prime \prime}$ latitud norte y $107^{\circ} 56^{\prime} 20.05^{\prime \prime}$ longitud oeste, a una altitud de $1420 \mathrm{msnm}$. El clima predominante es muy seco templado $\left[\mathrm{BWkw}\left(\mathrm{x}^{\prime}\right)\right]$ y la temperatura media anual es de $16.5{ }^{\circ} \mathrm{C}$ (Instituto Nacional de Estadística Geografía e Informática [INEGI], 2003). La precipitación media anual es de $312 \mathrm{~mm}$, con régimen de lluvias en verano y la precipitación invernal es de $10.2 \%$ del registro anual (INEGI, 2003). La evaporación total anual normal es de 1971 mm (Comisión Nacional del Agua [CONAGUA], 2020). El tipo de suelo es litosol + regosol eútrico de textura media (INEGI, 2003).

La plantación se realizó el 27 de julio de 2017 con planta de mezquite de seis meses de edad. La planta fue producida en bolsas negras de polietileno en un vivero de la región y el diámetro promedio de la planta fue de $3.8 \mathrm{~mm}$ $($ Desv. Est. $=0.7 \mathrm{~mm})$ con una altura promedio de $14.7 \mathrm{~cm}($ Desv. Est. $=3.0$ $\mathrm{cm})$. La planta fue podada en vivero. La plantación se realizó dentro de un área de restauración de mayor extensión, donde se construyeron bordos en curvas a nivel para retener escurrimientos e incrementar la humedad. La distancia entre bordos fue de $12 \mathrm{~m}$ y entre cada bordo se realizaron terrazas individuales para el establecimiento de las plantas. La excavación en la terraza fue de 30 $\mathrm{cm}$ de profundidad y con un diámetro aproximado de $60 \mathrm{~cm}$. La pendiente del terreno es alrededor del 3\%. Se utilizó un polímero hecho a base de potasio comercialmente conocido como Lluvia Sólida ${ }^{\circledR}$, que de acuerdo a su etiqueta comercial es capaz de adsorber hasta 400 veces su peso en agua, dependiendo del tipo de suelo, calidad del agua, clima, etc.

El diseño experimental fue completamente al azar con cuatro tratamientos (dosis de hidrogel por planta) y cinco repeticiones. Las unidades experimentales fueron líneas de 25 plantas dando un total de 500 plantas en todo el experimento. Las dosis fueron: $0 \mathrm{~g}$ (testigo), $3 \mathrm{~g}, 6 \mathrm{~g}$ y $9 \mathrm{~g}$ de hidrogel aplicado en seco. Estas dosis fueron inferiores a las recomendadas por los fabricantes, las cuales son alrededor de $20 \mathrm{~g}$ por planta. El hidrogel en seco fue mezclado con el suelo que rodea a la raíz de la planta. No se realizó la hidratación previa del hidrogel debido a la acumulación de agua en las terrazas individuales. Después de la plantación se realizaron evaluaciones de supervivencia el 30 de agosto de 2017, 11 de noviembre de 2017 y el 7 de junio de 2018. Se evaluó también la humedad del suelo (contenido volumétrico de agua) con un medidor portátil FieldScout TDR 300 (TimeDomain Reflectometer- Spectrum Technologies Inc) en las mismas fechas de sobrevivencia. Para ello se utilizaron varillas de $20 \mathrm{~cm}$ de largo que fueron introducidas en el suelo alrededor de la planta. La precipitación durante el periodo de estudio fue medida directamente en el sitio de plantación mediante 
un pluviómetro automático de balancín con data logger (HOBO®). No se realizaron riegos a la plantación.

La segunda plantación se estableció en el municipio de Aldama, Chihuahua. El sitio se localiza en las coordenadas $28^{\circ} 51^{\prime} 07.80^{\prime \prime}$ latitud norte y $105^{\circ} 52^{\prime}$ 07.08" longitud oeste, a una altitud de $1205 \mathrm{~m} \mathrm{snm}$. El clima predominante en la región es muy seco semicálido [BSOkw (w)] y la temperatura anual varía entre 18 y $22{ }^{\circ} \mathrm{C}$ (INEGI, 2003). La precipitación anual fluctúa entre 250 y $300 \mathrm{~mm}$, con régimen de lluvias en verano (Instituto Nacional de Investigaciones Forestales Agrícolas y Pecuarias [INIFAP], 2006). La precipitación invernal es menor al 5\% del registro anual y el tipo de suelo es Xerosol Lúvico de textura media (INEGI, 2003). Esta plantación se realizó el 9 de septiembre de 2017, prácticamente terminada la temporada de lluvias, esto con el fin evaluar la efectividad del hidrogel en condiciones de escaza humedad. Se utilizó planta de cuatro meses de edad, producida en vivero con malla sombra y en contenedores de polietileno negro, con un volumen de $310 \mathrm{~cm}^{3}$. El diámetro promedio de la planta fue de $3.8 \mathrm{~mm}$ (Desv. Est. $=0.3 \mathrm{~mm}$ ), con una altura promedio de $40.8 \mathrm{~cm}$ (Desv. Est. $=9.5 \mathrm{~cm}$ ). Para la preparación del terreno se utilizó una rastra de discos de forma uniforme en todo el terreno, de tal forma que se facilitara la realización de las cepas para la plantación. El terreno es plano y debido a la rugosidad creada por el paso de la rastra no hubo escurrimiento superficial hacia alguna dirección en particular, por lo que no fue necesario realizar terrazas individuales a las plantas o alguna otra obra de captación de agua.

El diseño experimental consistió en cuatro tratamientos distribuidos completamente al azar con cinco repeticiones. Las unidades experimentales fueron líneas de 10 plantas, dando un total de 200 plantas en todo el experimento. Las dosis fueron $0 \mathrm{~g}$ (testigo), $3 \mathrm{~g}, 6 \mathrm{~g}$ y $10 \mathrm{~g}$ del polímero retenedor de humedad, el cual en este caso fue previamente hidratado en dos litros de agua al momento de la plantación. A las plantas testigo se les aplicó solo agua. Después de la plantación se realizaron evaluaciones de supervivencia a los 5, 13, 19, 26, 37 y 45 días, así como a los $2.5,6.4$ y 9.5 meses. Se evaluó también la humedad del suelo con la sonda TDR 300 y se utilizó un pluviómetro automático para medir la precipitación en el sitio.

La tercera plantación fue establecida en la misma zona que la segunda plantación de Aldama, pero un año más tarde (01 de agosto de 2018) y con planta de mayor edad. Se utilizaron plantas de 15 meses de edad, producidas en bolsas de polietileno negro en un vivero con malla sombra. A diferencia de la plantación anterior la planta se estableció en plena temporada de lluvias con la finalidad de evaluar el efecto de las lluvias en la rehidratación del hidrogel. El diámetro promedio de la planta fue de $5.5 \mathrm{~mm}$ (Desv. Est. $=1.3 \mathrm{~mm}$ ), con una altura promedio de $39.7 \mathrm{~cm}$ (Desv. Est. $=11.9 \mathrm{~cm}$ ). La preparación del terreno fue similar a la segunda plantación. El experimento consistió en sólo 
dos tratamientos con cuatro repeticiones distribuidas completamente al azar. Los tratamientos fueron $0 \mathrm{~g}$ (testigo) y $10 \mathrm{~g}$ del polímero por planta, el cual fue previamente hidratado en dos litros de agua al momento de la plantación. A las plantas testigo se les aplicó solo agua. La unidad experimental fueron líneas de 26 plantas dando un total de 208 plantas en todo el experimento. Después de la plantación se realizaron evaluaciones de supervivencia a los 15 días, 5 , 10 y 12 meses. Se evaluó también la humedad del suelo alrededor de cada planta con la sonda TDR 300 en las mismas fechas de evaluación de sobrevivencia, además de otras tres ocasiones después de eventos grandes de lluvia. La precipitación fue medida con un pluviómetro automático en el sitio de plantación.

\section{Análisis de datos}

Se realizaron análisis de varianza de forma separada para cada fecha de medición y en cada sitio de plantación, a fin de evaluar el efecto de las diferentes dosis de hidrogel sobre la humedad del suelo y la supervivencia. Para la tercera plantación donde sólo hubo una dosis de hidrogel se realizaron pruebas de t-student. Todos los análisis y gráficas se realizaron mediante el Software R v. 3.6.1. (R Core Team, 2020). Las comparaciones múltiples de medias fueron realizadas mediante la prueba de Tukey con una diferencia significante a $\mathrm{p} \leq 0.05$. Los datos de precipitación diaria y por evento fueron procesados en el programa RIST (Rainfall Intensity Summarization Tool) versión 3.99 (United States Department of Agriculture [USDA], 2019).

\section{Resultados}

\section{Plantación en Nuevo Casas Grandes, Chih. 2017-2018}

El promedio de supervivencia en la primera evaluación realizada en Nuevo Casas Grandes, Chihuahua, el 30 de agosto de 2017 (un mes después de la plantación) fue de $67.8 \%$ (Desv. Est. = 8.7\%), sin diferencias significativas entre los tratamientos $(\mathrm{p} \geq 0.41)$. El testigo tuvo un porcentaje un poco más alto con $81 \%$ (Desv. Est. $=8.9 \%$ ), mientras que el resto de los tratamientos tuvieron una media entre 62 y $65 \%$ (Figura 1A). A casi cuatro meses de la plantación (Figura 1B), la supervivencia tuvo un ligero decremento en todos los tratamientos, con una media general de $64.0 \%$, y sin diferencias significativas entre tratamientos $(p \geq 0.5)$. El rango en las medias fue del 59 al $76 \%$, donde el testigo se mantuvo con el mayor porcentaje. A prácticamente un año después de la plantación la media en supervivencia decreció a $40 \%$ (Figura 1C), con un rango entre 36\% y $46 \%$, donde el valor mínimo de este rango correspondió a la dosis de $3 \mathrm{~g}$, y el valor máximo al testigo, sin diferencias significativas entre tratamientos $(\mathrm{p} \geq 0.6)$. 


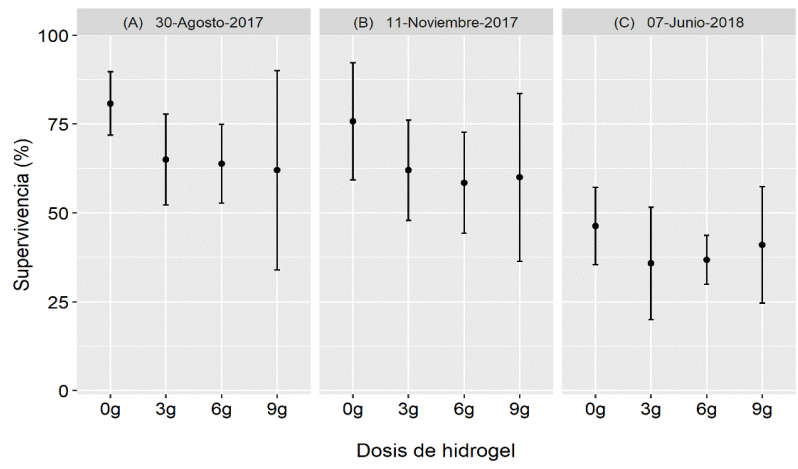

Figura 1. Supervivencia de mezquite (media \pm desviación estándar) con diferentes dosis de hidrogel por planta, en tres fechas de evaluación de la plantación en Nuevo Casas Grandes, Chihuahua. No se encontraron diferencias significativas dentro de cada fecha de evaluación.

La precipitación total entre la fecha de plantación y la última evaluación, es decir, a prácticamente un año, fue de sólo $142 \mathrm{~mm}$, lo cual es apenas el $45 \%$ de la precipitación media anual reportada en la región de estudio. Se registraron sólo dos eventos de precipitación importantes, de 20 mm de lluvia cada uno, al cuarto y quinto días posteriores a la fecha de plantación (Figura 2). Después de dichos eventos, las lluvias más significativas ocurrieron el 15 y 19 de agosto con 15 y $24 \mathrm{~mm}$, respectivamente. En seguida de estas fechas no hubo ningún evento que haya superado los $10 \mathrm{~mm}$ y no hubo precipitación después del 18 de noviembre de 2017.

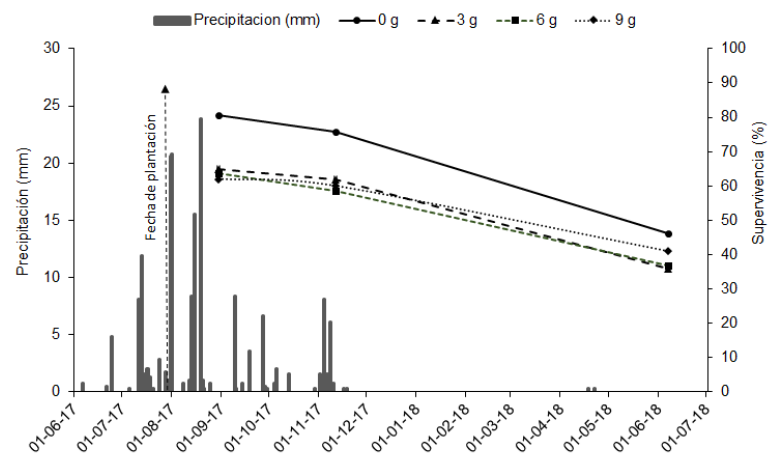

Figura 2. Precipitación por eventos de junio 2017 a junio 2018 y supervivencia de mezquite por dosis de hidrogel en Nuevo Casas Grandes, Chihuahua.

Los resultados de humedad en el suelo no mostraron diferencias estadísticamente significativas entre los tratamientos en ninguna de las evaluaciones. En la primera evaluación, a prácticamente un mes después de la plantación y 10 días después del último evento significativo de lluvia, el promedio general de humedad fue del $11.6 \%$ (Desv. Est.=1.4\%), con medias muy similares entre tratamientos $(\mathrm{p} \geq 0.46)$ (Figura 3 ). 


\section{Plantación en Aldama, Chih. 2017-2018}

La segunda plantación se realizó en Aldama el 9 de septiembre de 2017, prácticamente al término de la temporada de lluvias. Al momento de la plantación, la precipitación acumulada fue de $262 \mathrm{~mm}$ y los eventos más significativos de lluvia ocurrieron 15 días antes de la plantación (Figura 4). A partir de la fecha de plantación y hasta la última evaluación del siguiente año (2018), la precipitación total registrada fue de sólo $62 \mathrm{~mm}$ con muy pocos eventos de lluvia y distanciados en tiempo (Figura 4).

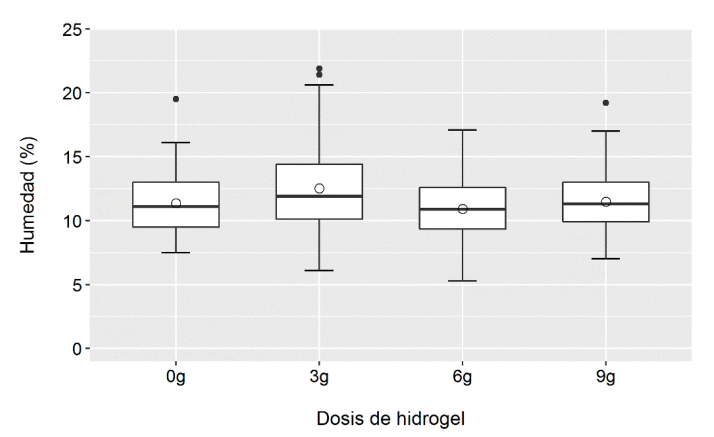

Figura 3. Porcentaje de humedad en el suelo por dosis de hidrogel a un mes de la plantación con mezquite en Nuevo Casas Grandes, Chihuahua. La línea horizontal dentro de la caja representa la mediana, el circulo el promedio, la caja contiene entre el $25 \%$ y $75 \%$ de los datos y los puntos son datos atípicos. $\mathrm{n}=125$ plantas por tratamiento. Las diferencias no fueron estadísticamente significativas.

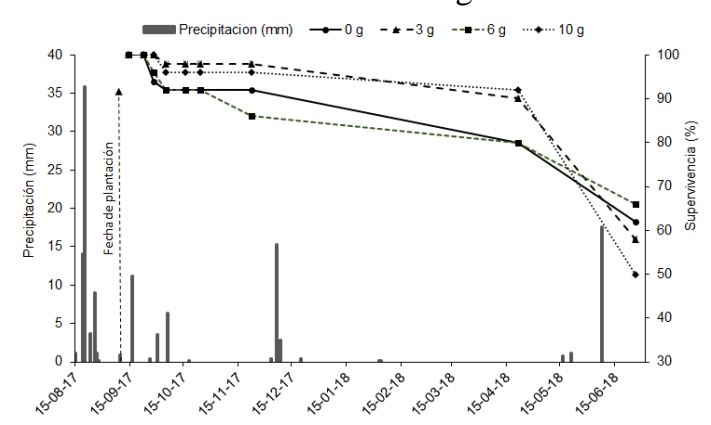

Figura 4. Precipitación por eventos de agosto 2017 a junio 2018 y supervivencia de mezquite con diferentes dosis de hidrogel en una plantación de 2017 en Aldama, Chihuahua.

Durante las primeras dos evaluaciones ( 5 y 13 días después de la plantación) no hubo mortandad de planta (Figura 5A, 5B). Sin embargo, la humedad en el suelo disminuyó drásticamente y sorpresivamente el promedio más alto de humedad a los cinco días después de la plantación fue para el testigo con $10.3 \%$ (Desv. Est.= 2.4\%) (Figura 6A). Este valor fue significativamente diferente sólo al tratamiento de $6 \mathrm{~g}$ por planta, que tuvo el valor de humedad más bajo con $7.6 \%$ (Desv. Est. $=0.7 \%)(p \geq 0.04)$. No hubo 
diferencia entre el testigo y los otros dos tratamientos: $3 \mathrm{~g}$ y $10 \mathrm{~g}$ por planta, cuyo promedio fue de $8.5 \%$ y $8.9 \%$, respectivamente.

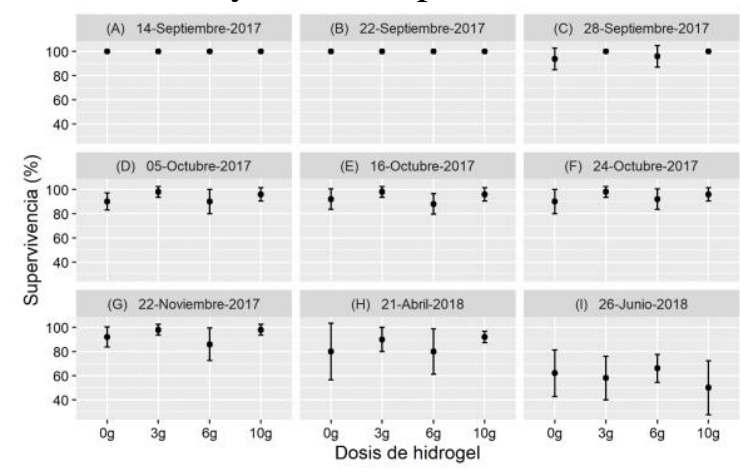

Figura 5. Promedio y desviación estándar por dosis de hidrogel, para nueve evaluaciones de supervivencia de mezquite en una plantación de 2017 en Aldama, Chihuahua. No se encontraron diferencias estadísticamente significativas entre los tratamientos en ninguna de las evaluaciones.

Posterior a la primera evaluación de humedad, el único evento de precipitación más significativo fue el día 16 de septiembre de 2017 con $11 \mathrm{~mm}$ de lluvia (Figura 4). Aparentemente, dicho evento no fue suficiente para la rehidratación del polímero, pues el segundo muestreo de humedad tuvo valores bastantes similares al primero (Figura 6B), con una media general de $8.4 \%$ (Desv. Est. $=2.8 \%$ ). Posterior a esta evaluación no se encontraron diferencias en el contenido de humedad en el suelo.

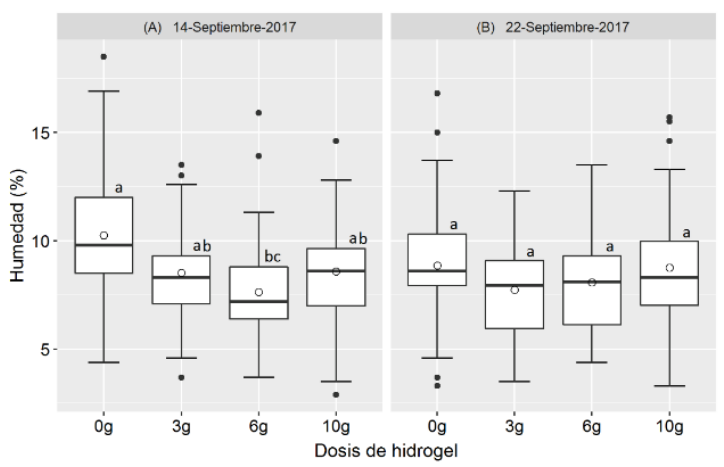

Figura 6. Porcentaje de humedad en el suelo, por dosis de hidrogel, a los 5 y 13 días después de la plantación de mezquite en 2017 en Aldama, Chihuahua. Letras diferentes indican diferencias significativas.

En cuanto a la supervivencia a los 19 días después de la plantación, la media general decreció al 98\% (Figura 5C), pero sin diferencias entre tratamientos ( $\mathrm{p} \geq 0.37$ ). Hasta los 2.5 meses después de la plantación (Figura $5 \mathrm{G})$, el porcentaje de supervivencia para todos los tratamientos se mantuvo entre 90 y $98 \%$, excepto para la dosis de 6 g por planta, que tuvo un promedio más bajo con $86 \%$, pero esta diferencia no fue significativa con respecto a los 
otros tratamientos ( $\mathrm{p} \geq 0.11$ ). Durante la época de sequía (abril de 2018, Figura $5 \mathrm{H}$ ), la supervivencia disminuyó a $86 \%$ y sin diferencia significativas entre tratamientos $(\mathrm{p} \geq 0.51)$. Los tratamientos de $3 \mathrm{~g}$ y $10 \mathrm{~g}$ tuvieron valores cercanos con una media de 90 y $92 \%$, mientras que el testigo tuvo un $80 \%$ de supervivencia y este valor fue igual a la media del tratamiento de $6 \mathrm{~g}$ por planta.

En la última evaluación y prácticamente después del periodo de sequía la media general disminuyó a 59\% y tampoco se presentaron diferencias significativas entre tratamientos $(\mathrm{p} \geq 0.56)$. El valor más bajo al final de la evaluación fue para el tratamiento de $10 \mathrm{~g}$ por planta con una media de $50 \%$, y la media incrementó a 58, 62 y $66 \%$ para los tratamientos de 3, 0 y 6 g, respectivamente (Figura 5I).

\section{Plantación en Aldama, Chih. 2018-2019}

La tercera plantación realizada el 01 de agosto de 2018 en Aldama mostró resultados similares a las anteriores, aunque con una ligera ventaja en el tratamiento de hidrogel con respecto al testigo, pero sin diferencias estadísticamente significativas (Figura 7).

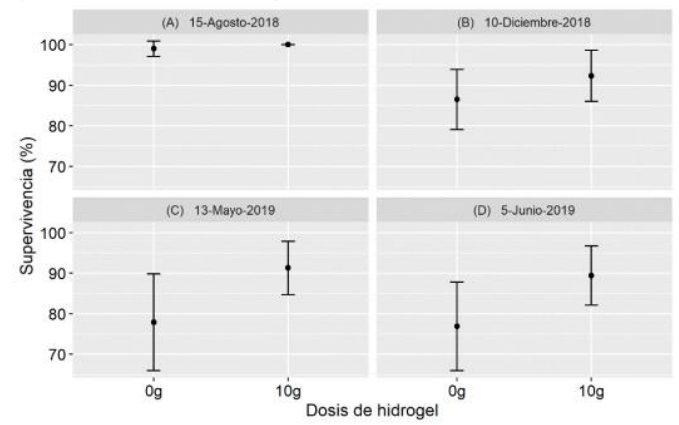

Figura 7. Promedio y desviación estándar por dosis de hidrogel, para cuatro evaluaciones de supervivencia de mezquite en una plantación de 2018 en Aldama, Chihuahua. No se encontraron diferencias estadísticamente significativas en ninguna de las evaluaciones.

A 15 días de la plantación prácticamente no hubo mortandad de planta (Figura 7A). La plantación además se benefició por un evento de lluvia de casi $60 \mathrm{~mm}$ el día 17 de agosto de 2018 (Figura 8), lo que permitió mantener un nivel alto de supervivencia. Después de este evento de precipitación se realizaron muestreos de humedad los días 24 y 27 de agosto para ver el efecto en la rehidratación del hidrogel, pero no se encontraron diferencias significativas ( $\mathrm{p}=0.32$ y $\mathrm{p}=0.57$ ) (Figura $9 \mathrm{~B}$ y $9 \mathrm{C}$ ). 


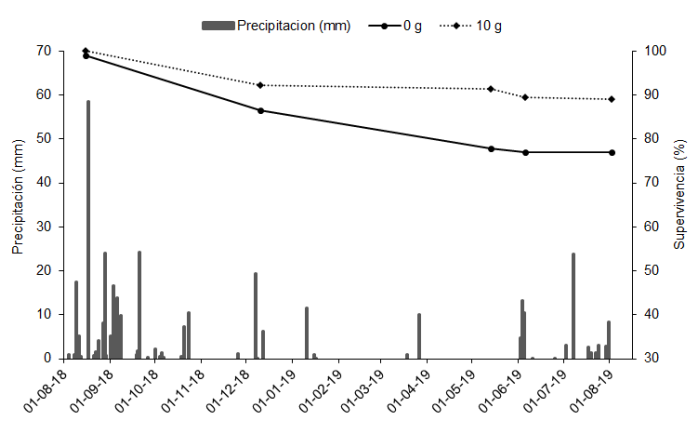

Figura 8. Precipitación por eventos de agosto 2018 a agosto 2019 y supervivencia de mezquite por dosis de hidrogel en la plantación de 2018 en Aldama, Chihuahua.

La segunda evaluación de supervivencia fue realizada el día 10 de diciembre de 2018 y la media en supervivencia para el testigo fue del $86.5 \%$, mientras que la media para el tratamiento con hidrogel fue de $92.3 \%$, sin diferencias significativas ( $\mathrm{p} \geq 0.27$ ) (Figura 7). Esta evaluación fue realizada tres días después de un evento de precipitación de $20 \mathrm{~mm}$ (Figura 8), sin embargo, no se encontraron diferencias en la humedad del suelo entre el tratamiento con hidrogel y el testigo (Figura 9D), cuyo promedio general fue de $13.5 \%$. La tercera evaluación en la supervivencia fue realizada en mayo de 2019, es decir, en las últimas semanas de la temporada de sequía y tampoco se encontraron diferencias significativas $(p \geq 0.09)$. Esto a pesar de que el testigo tuvo una reducción de $8.7 \%$ con respecto a la evaluación anterior, mientras que el tratamiento con hidrogel se redujo en sólo 1.0\% (Figura 7).

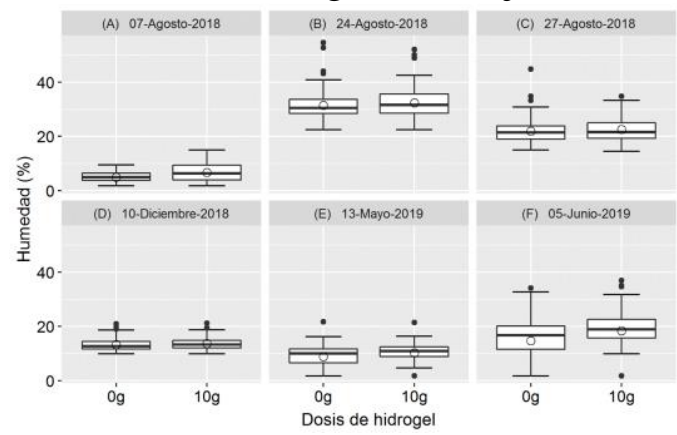

Figura 9. Contenido de humedad en el suelo en seis evaluaciones realizadas a la plantación con mezquite entre 2018 y 2019 en Aldama, Chihuahua.

La penúltima evaluación fue realizada el 5 de junio de 2019, después de dos eventos de precipitación los días 3 y 4 de junio, con 13 y $11 \mathrm{~mm}$ de lluvia respectivamente. Sin embargo, aparentemente esto no fue suficiente para la rehidratación del hidrogel pues no se encontraron diferencias significativas en la humedad ( $p \geq 0.76$ ) (Figura 9F). A partir de esta fecha inició el periodo de lluvias de 2019, por lo que no se encontraron diferencias significativas tanto en la supervivencia como en la humedad en la última 
evaluación del 01 de agosto de 2019, con una media general en la supervivencia del $83.2 \%$.

\section{Discusión}

Los resultados indican que no hubo efecto positivo de ninguna de las dosis de hidrogel en la supervivencia de mezquite. Esto puede ser explicado por una rápida deshidratación del hidrogel debido a las condiciones extremas del clima. Al respecto, otros estudios han indicado que los polímeros retenedores de humedad tienen poca efectividad para incrementar la supervivencia o crecimiento bajo condiciones extremas de sequía. Sloan (1994) sugiere que esto se debe a que la cantidad de agua absorbida por un hidrogel es suficiente para mantener una planta viva por un periodo de tiempo corto, pero es difícil mantener la supervivencia si las condiciones de sequía persisten. En un experimento de estrés hídrico con Pinus halepensis de seis meses de edad en macetas, Hüttermann et al. (1999) encontraron que las plantas tratadas con $2 \mathrm{~g}$ de hidrogel por cada kilogramo de suelo (Stockosorb ${ }^{\circledR}$ K400), retardaron la mortalidad de la planta por 6 días comparado al testigo, mientras que un mayor volumen de hidrogel $\left(4 \mathrm{~g} \mathrm{~kg}^{-1}\right)$ retardó la mortalidad por 18 días. En un estudio similar, Savé et al. (1995) encontraron que el uso de 200 y $400 \mathrm{ml}$ de hidrogel hidratado en macetas de 2.9 litros, retardó la mortalidad de Pinus pinea L. de un año de edad comparado con el testigo, por 10 y 28 días, respectivamente. Estos resultados, por lo tanto, indican que puede haber un beneficio en la supervivencia, siempre y cuando las condiciones extremas de sequía no se extiendan más allá del periodo de beneficio obtenido por el uso del hidrogel.

El beneficio temporal del hidrogel, sin embargo, puede ser nulo o bastante corto en zonas áridas dado los largos periodos sin precipitación y altas temperaturas. Los resultados de este estudio por ejemplo indican que el efecto temporal en la humedad puede ser incluso menor a cinco días en condiciones de muy baja humedad en el suelo (Figura 6). Sin embargo, la mayor mortandad de planta en los tres experimentos se registró durante los meses más críticos de sequía que son de marzo a junio, es decir, entre siete y diez meses después de la fecha de plantación. Esto se debe a que son los meses con prácticamente nula precipitación (Figura 2 y Figura 4) y máximas temperaturas. Los promedios mensuales de las temperaturas máximas en Aldama, Chihuahua entre abril y junio van de 25 a $32{ }^{\circ} \mathrm{C}$ (CONAGUA, 2020). Esta falta de precipitación y altas temperaturas explican la mayor mortandad de planta durante este periodo del año, meses en que ya no hay un efecto del hidrogel, independientemente de la dosis utilizada.

Otro aspecto importante es la rehidratación del polímero. Los resultados de este estudio indican que eventos de entre 10 y $20 \mathrm{~mm}$ no son suficientes para la rehidratación del hidrogel utilizado. Al respecto, Yu et al. 
(2011) encontraron que el tiempo de hidratación de un hidrogel es mucho más largo cuando está mezclado con el suelo, que cuando se encuentra en agua destilada. Crous (2017) explica que esto se debe a que el polímero sólo absorbe el agua que está en contacto directo con la partícula de hidrogel, y no es capaz de absorber agua de su alrededor cuando se encuentra mezclado con el suelo. En este sentido, Ghebru et al. (2007) recomiendan aplicar riegos al suelo provisto con hidrogel antes de que el suelo este completamente seco, con el fin de que el polímero pueda reabsorber agua eficientemente. El riego, sin embargo, es simplemente una actividad que no es considerada en las plantaciones de restauración forestal debido a la dificultad de transportar agua hasta los sitios de plantación y a los altos costos que esto implicaría.

Otro tema que aún es objeto de discusión es la dosis de hidrogel recomendada. Al respecto se han hecho muchos experimentos, pero la mayoría de ellos han sido con plantas en macetas y bajo condiciones controladas. Por ejemplo, en el estudio realizado por Hüttermann et al. (1999) mencionado anteriormente, se utilizaron dosis de 2 y $4 \mathrm{~g}$ de hidrogel por kilogramo de suelo en maceta. Si consideramos que la dimensión de una cepa común para la plantación de un árbol debe ser de 40 x 40 x $40 \mathrm{~cm}$ (CONAFOR, 2010), esto da un volumen de $64000 \mathrm{~cm}^{3}$. Asumiendo una densidad aparente del suelo de $1.2 \mathrm{~g} \mathrm{~cm}^{-3}$ tenemos $76.8 \mathrm{~kg}$ de suelo por cepa común, es decir, en una dosis de $10 \mathrm{~g}$ de hidrogel por planta se tiene una relación de $0.13 \mathrm{~g}$ de hidrogel por kilogramo de suelo. Esto es de 15 a 30 veces menos que aquellas dosis utilizadas por Hüttermann et al. (1999). Esto sin considerar que la cepa común no es un medio aislado del resto del suelo, pues en una plantación a una distancia de tres metros entre plantas (1111 árboles por hectárea), la planta es expuesta a un volumen de suelo mucho mayor. Todo este suelo que rodea a la planta hace que la humedad provista por el hidrogel sea expuesta a un alto potencial de succión, dado por la absorción y capilaridad del potencial matricial del suelo, y el potencial osmótico, mismo que puede ser muy alto en caso de suelos salinos (Jury \& Horton, 2004). Además de esto, la humedad inyectada al suelo mediante el hidrogel también puede ser afectada por el posible flujo gravitacional del agua al momento de la aplicación, así como la disminución de humedad por el agua que toman las plantas para sobrevivir a la sequía. Con todas estas consideraciones no es de extrañarse que el hidrogel dura poco tiempo hidratado en zonas áridas.

En cuanto a la forma de aplicación, se ha reportado que aplicaciones de hidrogel en seco justo por debajo de la raíz de la planta pueden resultar contraproducente, ya que al expandirse el hidrogel la planta es sacada del suelo (Sarvas et al., 2007). En este estudio no se observó este problema debido a que la aplicación de hidrogel en seco se mezcló con el suelo. Es importante también considerar que la aplicación en seco se realizó sólo en la plantación donde se realizaron terrazas individuales a la planta. Es decir, el suelo debe 
llegar a saturarse completamente de agua para asegurar la hidratación del hidrogel. Por otro lado, se han realizado experimentos cubriendo la raíz de la planta con hidrogel hidratado antes de plantar (Sloan 1994), pero los resultados no han sido satisfactorios debido al espacio poroso que dejan las partículas de hidrogel una vez deshidratadas, lo que causa espacios de aire alrededor de las raíces más finas. Un efecto similar fue observado en las dosis más altas en este estudio. Observaciones cualitativas al momento de incrustar las varillas del TDR indicaron que el suelo estaba más poroso alrededor de las plantas con mayor dosis, en comparación al testigo. Esto podría explicar los valores más altos de humedad para el testigo (Figura 6), dado que las varillas del TDR estuvieron menos expuestas a espacios de aire en el suelo al momento de tomar la lectura. Otra observación que refuerza esta hipótesis son pequeños hundimientos de suelo alrededor de las plantas con las dosis más altas de hidrogel (Figura 10).

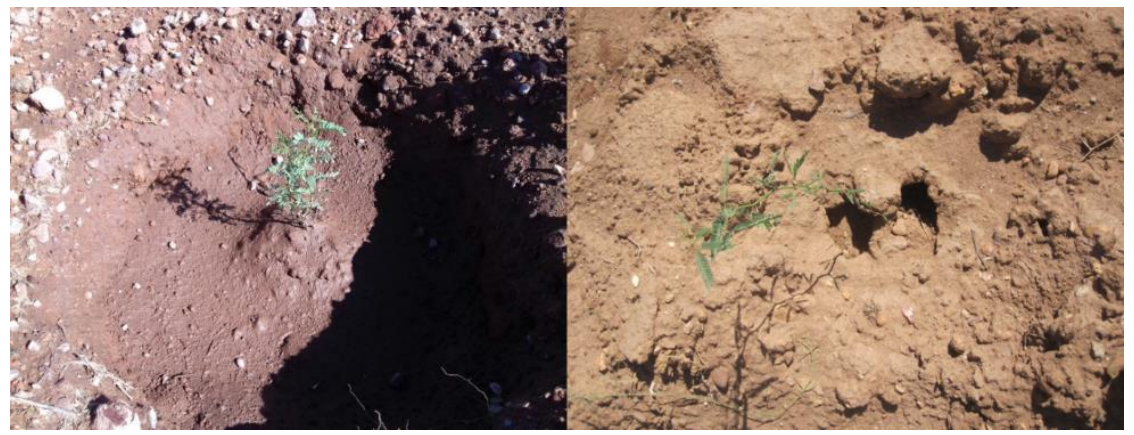

Figura 10. Planta de mezquite con nueve gramos de hidrogel mostrando un ligero hinchamiento en el suelo por efecto de la hidratación del hidrogel, después de un evento de lluvia (izquierda). Planta con hundimientos en el suelo al momento de tomar las lecturas de humedad con el TDR, debido a la alta porosidad creada por las partículas del hidrogel deshidratado (derecha).

Estos hundimientos pueden ser explicados por los espacios porosos que deja el hidrogel en el suelo una vez deshidratado. De la misma forma, esto explicaría la alta variabilidad que se encontró en los porcentajes de supervivencia de la dosis de $9 \mathrm{~g}$ en la plantación de 2017 en Aldama (Figura 1). Es decir, entre más alta sea la dosis, hay más probabilidad de que la raíz de la planta quede expuesta a más espacios con aire debido a la mayor cantidad de partículas de hidrogel.

Otros estudios en México con dosis más altas tampoco han reportado beneficios significativos en la supervivencia. En un estudio donde se utilizó hidrogel para el establecimiento de Pinus greggi en Coahuila (García, 2017), no se encontraron diferencias significativas en la humedad entre una dosis de $25 \mathrm{~g}$ de hidrogel por planta (40\% de humedad) y el testigo (33\% de humedad) durante 10 meses de la plantación. De igual forma, no se reportó una diferencia significativa entre la supervivencia, con una media de $83 \%$ para el tratamiento 
de hidrogel, contra $92 \%$ en el testigo. En otro estudio sobre supervivencia y desarrollo de Pinus arizonica bajo condiciones de sequía extrema en Coahuila, tampoco encontraron diferencias estadísticamente significativas en la supervivencia entre las dosis de $2.5,5$ y $7.5 \mathrm{~g}$, con una media general de $43 \%$ (Patricio, 2014). En general este y varios estudios indican que la aplicación de hidrogeles en el ámbito de plantaciones de restauración forestal debe ser tomado con cautela, debido a los altos costos que implica y pocos o nulos beneficios que pueden ser obtenidos.

\section{Conclusiones}

El estudio evaluó el efecto de un polímero retenedor de humedad en la supervivencia de tres plantaciones de Prosopis glandulosa Torr. Los resultados indicaron que no hubo efecto positivo de ninguna de las dosis de hidrogel en la supervivencia. Estos resultados corresponden a zonas semiáridas con precipitaciones anuales menores a $300 \mathrm{~mm}$, plantaciones sin riego y dosis de hidrogel entre 3 y 10 g por planta. La efectividad del polímero para retener la humedad en el suelo es de muy corto tiempo y no tiene efecto durante los meses críticos de sequía y altas temperaturas, en los cuales se registró la mayor mortandad de planta. Esto indica que dosis más altas tampoco tendrían mayor efectividad en la supervivencia. La cantidad de precipitación de los pocos eventos de lluvia separados en tiempo no es suficiente para la rehidratación del polímero. Por consiguiente, los resultados sugieren que el incremento en la supervivencias de una plantación de restauración forestal con mezquite en zonas semiáridas no se consigue con la aplicación de hidrogel y sigue basado en los factores tradicionales que son, entre otros; calidad de planta, preparación del terreno y precipitación.

\section{References:}

1. Abedi-Koupai, J., Sohrab, F., \& Swarbrick, G. (2008). Evaluation of hydrogel application on soil water retention characteristics. Journal of plant nutrition, 31(2), 317-331. doi: 10.1080/01904160701853928

2. Al-Humaid, A. I., \& Moftah, A. E. (2007). Effects of hydrophilic polymer on the survival of buttonwood seedlings grown under drought stress. Journal of Plant Nutrition, 30(1), 53-66. doi:10.1080/01904160601054973

3. Andry, H., Yamamoto, T., Irie, T., Moritani, S., Inoue, M., \& Fujiyama, H. (2009). Water retention, hydraulic conductivity of hydrophilic polymers in sandy soil as affected by temperature and water quality. Journal of Hydrology, 373(1-2), 177-183. doi:10.1016/j.jhydrol.2009.04.020

4. Bai, W., Zhang, H., Liu, B., Wu, Y., \& Song, J. (2010). Effects of super-absorbent polymers on the physical and chemical properties of 
soil following different wetting and drying cycles. Soil use and management, 26(3), 253-260. doi:10.1111/j.1475-2743.2010.00271.x

5. Capó, A. (2001). Establecimiento de plantaciones forestales: Los ingredientes del éxito. Saltillo Coahuila: Universidad Autónoma Agraria Antonio Narro. Departamento Forestal.

6. Cisneros-Zayas, E., Cun-González, R., Rosales-Naranjo, L., \& González-Morales, D. (2018). Lluvia sólida, para un uso eficiente del agua. Resultados preliminares. Revista Ingeniería Agrícola, 8(1), 1320.

7. Comisión Nacional del Agua [CONAGUA]. (2020). Normales climatológicas por estado. Recuperado de https://smn.conagua.gob.mx/es/informacion-climatologica-porestado? estado $=$ chih

8. Comisión Nacional Forestal [CONAFOR]. (2016). Monitoreo de Restauración Forestal y Reconversión Productiva 2014. Zapopan, Jalisco, México: CONAFOR.

9. Comisión Nacional Forestal [CONAFOR]. (2010). Prácticas de reforestación. Zapopan, Jalisco, México: CONAFOR.

10. Cortés, A. B., Ramírez, I. X. B., Eslava, L. F. B., \& Niño, G. R. (2007). Evaluación de hidrogeles para aplicaciones agroforestales. Ingeniería e Investigación, 27(3), 35-44.

11. Crous, J. W. (2017). Use of hydrogels in the planting of industrial wood plantations. Southern Forests: a Journal of Forest Science, 79(3), 197-213. doi:10.2989/20702620.2016.1221698

12. Del Campo, A. D., Hermoso, J., Flors, J., Lidón, A., \& MavarroCerrillo, R. M. (2011). Nursery location and potassium enrichment in Aleppo pine stock 2. Performance under real and hydrogel-mediated $\begin{array}{lllll}\text { drought conditions. } & \text { Forestry, } 84 \text { (3), } 245 .\end{array}$ doi:10.1093/forestry/cpr009

13. Freitas, T. L., Bertonha, A., \& Andrade, A. C. (2002). Uso de hidrogel na agricultura. Revista do Programa de Ciências Agro-Ambientais, Alta Floresta, 1(1), 23-31.

14. García, V. J.T. (2017). Sobrevivencia y crecimiento en una plantación de Pinus greggii Engelm. establecida con retenedores de humedad en Saltillo, Coahuila, México. Tesis de Licenciatura, UAAAN, Saltillo, Coahuila, México.

15. Ghebru, M.G., Du Toit, E.S., \& Steyn, J.M. 2007. Water and nutrient retention by AquaSoil ${ }^{\circledR}$ and Stockosorb ${ }^{\circledR}$ polymers. South African Journal of Plant and Soil 42(1), 32-36. doi:10.1080/02571862.2007.10634778

16. Hüttermann, A., Zommorodi, M., \& Reise, K. (1999). Addition of hydrogels to soil for prolonging the survival of Pinus halepensis 
seedlings subject to drought. Soil and Tillage Research 50: 295-304. doi:10.1016/S0167-1987(99)00023-9

17. Instituto Nacional de Estadística Geografía e Informática [INEGI]. (2003). Síntesis de Información Geográfica del Estado de Chihuahua. Aguascalientes, México: INEGI.

18. Instituto Nacional de Investigaciones Forestales Agrícolas y Pecuarias [INIFAP]. (2006). Estadísticas Climatológicas del Estado de Chihuahua (Periodo 1961-2003). Chihuahua, México: Centro de Investigación Norte Centro. Libro Técnico No. 1.

19. Jury, W. A., \& Horton, R. (2004). Soil physics. John Wiley \& Sons.

20. Kazanskii, K. S., \& Dubrovskii, S. A. (1992). Chemistry and physics of "agricultural" hydrogels. Advances in Polymer Science, 104, 97133.

21. Mexal, J. G., \& Landis, D. T. (1990). Target seedling concepts: height and diameter. In: Rose, R., Campbell, S. J., \& Landis, T. D. (eds.). Target Seedling Symposium: Proceedings, Combined Meeting of the Western Forest Nursery Associations (pp. 17-35). Fort Collins, CO. USA: USDA Forest Service, Rocky Mountain Forest and Range Experiment Station.

22. Patricio, H. N. (2014). Evaluación de tres dosis de hidrogel en la sobrevivencia y desarrollo de Pinus arizonica Engelm. Variedad stormiae Martinez plantados bajo condiciones de sequía extrema. Tesis de licenciatura. UAAAN, Saltillo, Coahuila, México.

23. Prieto, R. J. A., Vera, C. G., \& Merlín, B. E. (2003). Factores que influyen en la calidad de brinzales y criterios para su evaluación en vivero. Durango, Dgo. México: Campo Experimental Valle del Guadiana-INIFAP-SAGARPA. Folleto Técnico Núm. 12.

24. R Core Team (2020). R: A language and environment for statistical computing. R Foundation for Statistical Computing, Vienna, Austria. Recuperado de https://www.R-project.org/.

25. Rodríguez-Trejo, D. A. (2006). Notas sobre el diseño de plantaciones de restauración. Revista Chapingo Serie Ciencias Forestales y del Ambiente 12(2), 111-123.

26. Rodríguez-Trejo, D. A. (2008). Indicadores de calidad de planta forestal. México, D. F. México: Mundi-Prensa.

27. Rojas, B., Aguilera, R., Prin, J.L., Cequea, H., Cumana, J., Rosales, E., \& Ramírez, M. (2004). Estudio de la germinación de semillas de tomate en suelos áridos extraídos de la península de Araya (Venezuela) al utilizar polímeros de tipo hidrogeles. Revista Iberoamericana de Polímeros, 5(1): 17-27.

28. Rivera-Hincapié, C. A., Baeza-Aragón, C. A., \& ChavarriagaMontoya, W. (2007). Efecto de un retenedor de agua y dosis crecientes 
de fertilizantes foliares sobre la producción de tomate chonto y larga vida bajo cubierta plástica agroclear. Agronomía, 15(1), 103-119.

29. Sarvas, M., Pavlenda, P., \& Takácová, E. (2007). Effect of hydrogel application on survival and growth of pine seedlings in reclamations. Journal of Forest Science, 53(5), 204-209.

30. Savé, R., Pery, M., Marfá, O., \& Serrano, L. (1995). The effect of a hydrophilic polymer on plant water status and survival of transplanted pine seedlings. HortTechnology 5(2): 141-143.

31. Sloan, J. P. (1994). The use of root dips on North American conifer seedlings: A review of the literature. Tree Planter's Notes 45(1): 2631.

32. United States Department of Agriculture [USDA]. (2019). RIST (Rainfall Intensity Summarization Tool). Recuperado de https://www.ars.usda.gov/southeast-area/oxford-ms/nationalsedimentation-laboratory/watershed-physical-processesresearch/research/rist/rist-rainfall-intensity-summarization-tooldownload/

33. Viero, P. W. M., Chiswell, K. E. A., \& Theron, J. M. (2002). The effect of a soil-amended hydrogel on the establishment of a Eucalyptus grandis clone on a sandy clay loam soil in Zululand during winter. The Southern African Forestry Journal, 193(1), 65-75. doi:10.1080/20702620.2002.10433519

34. Viero, P. W., \& Little, K. M. (2006). A comparison of different planting methods, including hydrogels, and their effect on eucalypt survival and initial growth in South Africa. Southern African Forestry Journal, 208(1), 5-13. doi:10.2989/10295920609505256

35. Yu, J., Shainberg, I., Yan, Y. L., Shi, J. G., Levy, G. J., \& Mamedov, A. I. (2011). Superabsorbents and semiarid soil properties affecting water absorption. Soil Science Society of America Journal 75(6): 2305-2313. doi:10.2136/sssaj2010.0397 